\title{
A bio-inspired computing model as a new tool for modeling ecosystems:The avian scavengers as a case study
}

\author{
M. Àngels Colomer ${ }^{\mathrm{a}}$, Antoni Margalida ${ }^{\mathrm{b}}$, Delfí Sanuy ${ }^{\mathrm{c}}$, Mario J. Pérez-Jiménez $^{\mathrm{d}}$ \\ a Department of Mathematics, University of Lleida, Av. Alcalde Rovira Roure, 191. 25198 Lleida, Spain \\ b Bearded Vulture Study and Protection Group, Apdo. 43, 25520 El Pont de Suert Lleida, Spain \\ ' Department of Animal Production, University of Lleida, Av. Alcalde Rovira Roure, 191. 25198 Lleida, Spain \\ ¿ Research Group on Natural Computing, Dpt. of Computer Science and Artificial Intelligence, University of Sevilla, Avda. Reina Mercedes s/n, 41012 Sevilla, Spain
}

Keywords:

P systems

Ecosystem

Avian scavengers

Conservation

\begin{abstract}
A B S T R A C T
The models used for ecosystems modeling are generally based on differential equations. However, in recent years new computational models based on biological processes, or bioinspired models, have arisen, among which are $P$ systems. These are inspired by the functions of cells and present important advantages with respect to traditional models, such as a high computational efficiency, modularity and their ability to work in parallel. They are simple, individual-based models that use biological parameters that can be obtained experimentally. In this work, we present the framework for a model based on P systems applied to the study of an ecosystem in which three avian scavengers (predators) interact with 10 wild and domestic ungulates (preys). The computation time for 100 repetitions, corresponding to 14 simulation years each, with an initial population composed of 385,422 individuals, was $30 \mathrm{~min}$. Our results suggest that the model presented, based on P systems, correctly simulates the population dynamics in the period of time analyzed. We discuss the usefulness of this tool in simulating complex ecosystems dynamics to aid managers, conservationists and policy-makers in making appropriate decisions for the improvement of management and conservation programs.
\end{abstract}

\section{Introduction}

Mathematical models describing predator-prey relationships are used to study dynamics between two populations when one of them depends on the other for food and survival. The relationships among species, particularly vertebrates, in asymmetrical intraguild predator systems are complex, and generalizations remain elusive (Litvaitis and Villafuerte, 1996), but have important implications for conservation biology (Polis and Holt, 1992). Regarding the use of management and conservation measures for endangered species, it is particularly important to have an available model that allows us to reliably predict the dynamics of such populations, especially of the most endangered species (e.g., Meretsky et al., 2000; Ortega et al., 2009; Oro et al., 2008; Chapron et al., 2009; Grande et al., 2009). This tool can be useful in decision-making and in optimizing management of the given ecosystem.

There are many processes in an ecosystem that run in parallel and are interrelated, hence it is essential to be able to organize these interrelations in a schematic and graphic way. Food webs are characterized by many weak interactions and a few strong interactions, which appear to promote community persistence and stability (Bascompte et al., 2005). In this sense, quantification of the strength of interactions between species is essential for understanding how ecological communities are organized and how they respond to human exploitation.

Generally, the tools used for ecosystems modeling are based on differential equations. For example, the Lotka-Volterra model has been one of the most frequently used for the modeling of two species, predator and prey (Murray, 2002), and Verhulst's model (logistic differential equations) has been utilized as a fundamental growth model in ecological studies because of its mathematical simplicity and single biological definition (Sakanoue, 2007, 2009). Fuzzy set theory has been used to estimate the parameters of the models based on differential equations studying the interaction between a prey and its predator (Da Silva et al., 2008). Despite the satisfactory results presented by these models, some authors question the deterministic and continuous approach they imply. Russell et al. (2009), for example, developed a stochastic model for three species by using ordinary logistic differential equations analyzed through numerical analyses techniques.

Viability models do not consider optimal solutions but rather define all possible evolutions of a dynamic system under given constraints (Mullon et al., 2004). Viability theory considers that 
the evolution of a system is non-deterministic but belongs to a set of possibilities, depending on its state. Due to the mathematical complexity, the implementation requires a system of low dimensionality ( $<4$ interacting components), which limits its effectiveness given the complexity of ecological models. Recently, some more complex models have arisen (i.e., taking into account a greater number of parameters and variables), that are difficult to calibrate and validate. Moreover, their application requires division into smaller sizes defined arbitrarily and approximating the global model (e.g., Fulton et al., 2003, 2004). In this sense, Lawrie and Hearne (2008) propose a non-arbitrary algorithm for the division of these large models.

Membrane Computing is an emergent branch of Natural Computing (Păun, 1998; Ciobanu et al., 2006; Păun et al., 2010) that was introduced with the purpose of defining computing devices, called $P$ systems, which abstract from the structure and the function of the living cells. Rather than being an alternative to more classical modeling frameworks, such as ODEs (Ordinary Differential Equations), P systems constitute a complementary approach to be used when the classical modeling approaches fail. The most important property of these models is their capacity to work in parallel and to capture the randomness of the natural environmental processes using stochastic strategies based on Gillespie's theory of stochastic kinetics (Gillespie, 1976, 1977) and the semantics defined by using probabilistic functions (Cardona et al., 2009, 2010b).

In previous works we tested the utility of this new framework to managers and conservationists by applying these models on a community of scavengers (Cardona et al., 2009, 2010a) and the Zebra mussel Dreissena polymorpha (Cardona et al., 2010b). Here, taking a scavenger community that depends on carrion provided by wild and domestic ungulates as a model, we introduce a new model based on P systems generalizing the previous models and enabling the simultaneous analysis of some interrelated trophic chains. First, interactions among species are shown by means of Networks. P systems associate a rule to each interaction observed in the network quantifying the existent interaction. In order to evaluate its robustness, the model is checked and validated by using the experimental information from the ecosystem, which corresponds to a period of 14 years, taking three avian scavengers (as predator species) and ten ungulate species (as preys) as case studies.

We discuss the results obtained from a methodological pointof-view and the usefulness of this tool in simulating complex ecosystems dynamics to aid managers, conservationists and policymakers in making appropriate decisions for the improvement of management and conservation programs.

\section{Material and methods}

\subsection{Ecosystem to be modeled}

The study was carried out in the Pyrenean and Prepyrenean mountains of Catalonia (NE Spain, Fig. 1). The ecosystem to be modeled is composed of 13 species: three avian scavengers (the Bearded vulture Gypaetus barbatus, the Egyptian vulture Neophron percnopterus and the Griffon vulture Gyps fulvus) as predator species, and six wild ungulates (the Pyrenean chamois Rupicapra pyrenaica, the Red deer Cervus elaphus, the Fallow deer Dama dama, the Roe deer Capreolus capreolus, the Wild boar Sus scrofa and the Mouflon Ovis orientalis) and four domestic ungulates that are found in an extensive or semi-extensive regime (the sheep Ovis aries, the goat Capra hircus, the cow Bos taurus and the horse Equus caballus) providing carrion for the avian scavengers and considered as prey species. Prey species are herbivores and their remains form the primary food resource for the avian scavengers in the study area $(>80 \%$ of the diet is based on these species, see Donázar, 1993; Margalida et al., 2009).

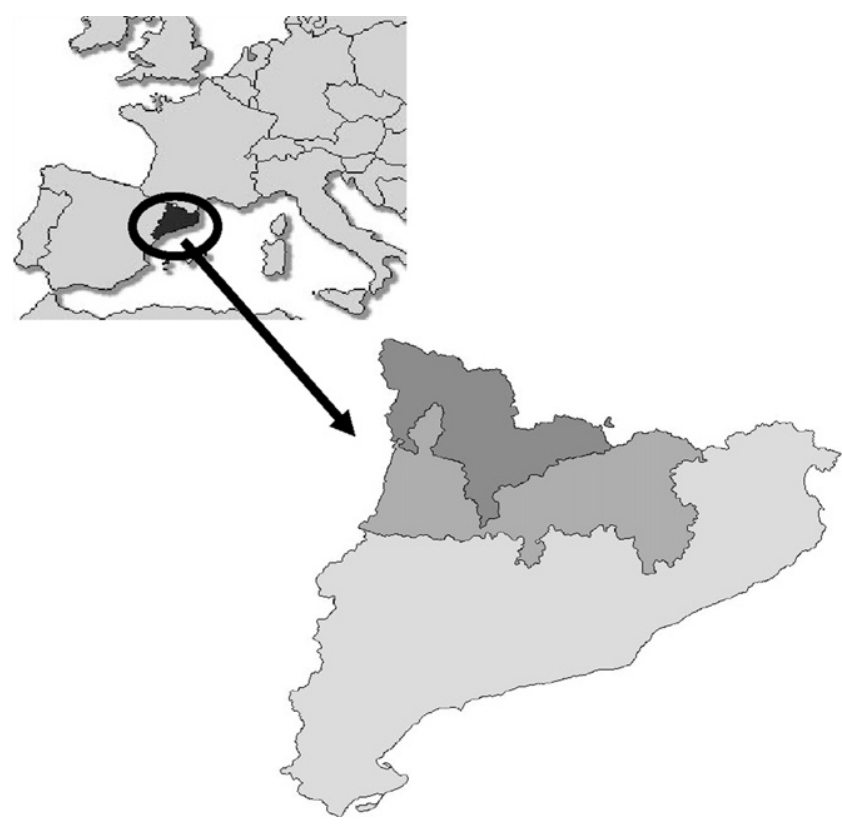

Fig. 1. Study area and subpopulations considered in the ecosystem. Pyrenees (dark grey); Prepyrenees (light grey).

The study area is characterized by the presence of two differentiated subpopulations that are interconnected. The Pyrenees region $\left(57,244 \mathrm{~km}^{2}\right)$ is characterized by the presence of abundant wild ungulates throughout the year, a strong presence of domestic ungulates during the summer (as a consequence of transhumance) and low human density. In the Pyrenean region, the level of annual rainfall ranges from 800 to $1200 \mathrm{~mm}$ and the maximum average temperatures do not exceed $25^{\circ} \mathrm{C}$ in the summer and do not fall under $-5^{\circ} \mathrm{C}$ in the winter. The orography presents altitudinal zones between 1000 and $3000 \mathrm{~m}$ where alpine terrain, which is characterized by the presence of meadows above $2200 \mathrm{~m}$, dominates and subalpine ground is characterized by wooded formations of Mountain pine (Pinus uncinata). Under $1600 \mathrm{~m}$, montane terrain is found where wooded formations are dominated by European beech (Fagus sylvatica). The Prepyrenean region $\left(77,372 \mathrm{~km}^{2}\right)$ is more populated by humans, with a more abundant domestic ungulate population that is regular during the winter, with low densities of wild ungulates. In the Prepyrenean region, the level of annual rainfall ranges from 800 to $1200 \mathrm{~mm}$ and maximum average temperatures do not exceed $30^{\circ} \mathrm{C}$ in the summer or fall under $-2{ }^{\circ} \mathrm{C}$ in the winter. The orography presents altitudinal zones between 600 and $1500 \mathrm{~m}$ corresponding to submontane ground, which is dominated in its lower area by Portuguese oak (Quercus faginea) and Holm oak ( $Q$. ilex sp. ballota) woods, and montane ground which is dominated by Downy oak (Q. pubescens). Occasionally, some mountain ranges are found in the montane region reaching $2000 \mathrm{~m}$. In regards to the distribution of the scavenger species, the bearded vulture population in the Pyrenees vs. Prepyrenees is $37.8 \%$ vs. $62.2 \%(n=37)$, the Egyptian vulture $10.2 \%$ vs. $89.8 \%(n=59)$ and the Griffon vulture $21.5 \%$ vs. $78.5 \%(n=822)$. Since individuals can move from one area to another according to the resources available, the ecosystem would function as a single set (global ecosystem) composed of two subsets (subpopulations separated by biogeographical criteria). In this way, whenever there is a lack of trophic resources in one of the subareas, the individuals can move to the other one. In both, the ecosystem load capacity has been limited to the appropriate areas and habitats for the different species as well as the maximum density that can be reached (see Appendix A).

The three scavenger species are cliff-nesting and only the Egyptian vulture is migratory (their presence in the study area is limited 


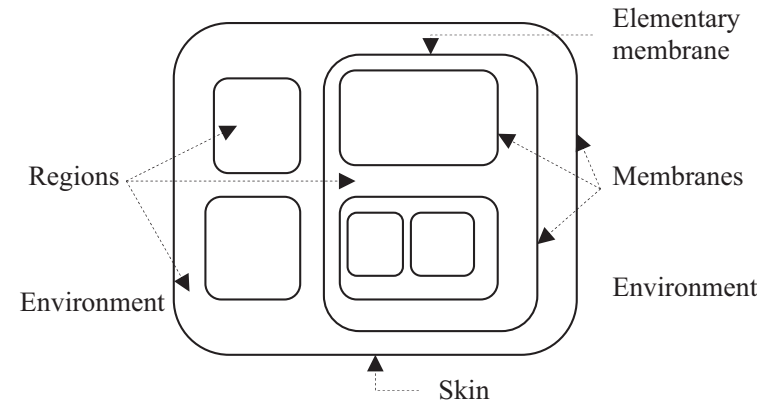

Fig. 2. Representation of a membrane structure.

to March-August). Concerning their trophic ecology, the diet of the Bearded vulture is based principally on bone remains of wild and domestic ungulates (principally sheep and Pyrenean chamois), although during the chick-rearing period small animals are important for the energetic requirements of the chicks (Margalida et al., $2005,2009)$. The foraging areas are about $20 \mathrm{~km}$ around the nest (A.M. unpubl. data). The Griffon vulture feeds mainly on wild and domestic ungulates and meat remains provided by sheep, pigs, cows and horses (Donázar, 1993). Grazing areas have a radius of approximately $25 \mathrm{~km}$ around the hill. Finally, the Egyptian vulture is a more opportunistic species having a more heterogeneous diet which is based mainly on small corpses of mammals and birds. It is more dependent on rubbish dumps and supplementary feeding areas, and grazing areas are limited to radii under $10 \mathrm{~km}$ from the nest (Donázar, 1993).

Concerning prey species, domestic ungulates are present in the Pyrenean subpopulation during the summer (May-October) as a consequence of transhumant movements and become food resources for avian scavengers during this period (see Olea and Mateo-Tomás, 2009) whereas in the Prepyrenean region, they are present throughout the year in an extensive or semi-extensive regime. Wild ungulates are present in the area all year with the densities of Pyrenenan chamois, Red deer and Mouflon being more important in the Pyrenean region and Wild boar more important in the Prepyrenean region (see Appendix A).

\subsection{A P system based model for ecosystems}

In this section, we will present the semantics and syntax used to model ecosystems by means of P systems and the specific model proposed for the ecosystem for the avian scavengers.

\subsubsection{P systems}

The starting point of this new model of computation is the observation that the cell is the smallest living thing as well as a tiny machine with a complex structure, and the assumption that the processes taking place in the compartmental structure of a living cell can be interpreted as computations. The challenge is to take the cell itself as a support for computations, and to find those elements useful for computations in the structure and the functioning of the cell as a whole. The devices of this model are called P systems, consisting of a cell-like membrane structure, in the compartments of which one places multisets of objects that evolve according to given rules.

The main components of P systems are the membrane structure, multisets of objects, and evolution rules (Fig. 2).

- A membrane structure consists of several membranes arranged in a hierarchical structure inside a main membrane (the skin), and delimiting regions (the space in-betweena membrane and the immediately inner membranes, if any). Each membrane identifies a region inside the system.

- Regions defined by a membrane structure contain objects corresponding to chemical substances present in the compartments of a cell. The objects can be described by symbols or by strings of symbols, in such a way that multisets of objects are placed in regions of the membrane structure.

- The objects can evolve according to given evolution rules, associated with the regions (and hence, with the membranes).

The functioning of a P system is defined as follows:

- A configuration of a cell-like membrane system consists of a membrane structure and a family of multisets of objects associated with each region of the structure. At the beginning, there is a configuration called the initial configuration of the system.

- In each time unit, we can transform a given configuration to another configuration by applying the evolution rules to the objects placed inside the regions of the configurations, in a nondeterministic, maximally parallel way (the rules are chosen in a non-deterministic way, and in each region, all objects that can evolve must do so). In this way, we get transitions from one configuration of the system to the next.

- A computation of the system is a sequence of configurations such that each one is obtained from the previous one by a transition, and shows how the system is evolving.

The approach has a series of features that overcome several drawbacks of classical models based on differential equations: modularity (intrinsic to a membrane system), scalability/extensibility (further membranes and/or further evolution rules can be added without essentially changing the way a system works), understandability (evolution rules directly correspond to chemical reactions or interactions among species), programmability (a rewriting-based model can be easily transformed into a program, with certain programming languages, such as JAVA, C++, CLIPS), while preserving other desirable features of differential equations models, such as non-linearity of evolution.

\subsubsection{The formal model}

In this section, we define a P system based framework where additional features, such as probabilistic functions and three electrical charges that better describe specific properties, are used.

A skeleton of an extended $P$ system with active membranes of degree $q \geq 1, \Pi=(\Gamma, \mu, R)$, can be viewed as a set of (polarized) membranes hierarchized by a structure of membranes $\mu$ (a rooted tree) labeled by $0,1, \ldots, q-1$. All membranes in $\mu$ are supposed to be (initially) neutral and they have associated with them $R$, a finite set of evolution rules of the form $u[v]_{i}^{\alpha} \rightarrow u^{\prime}\left[v^{\prime}\right]_{i}^{\beta}$ that can modify their polarization but not their label. $\Gamma$ is an alphabet that represents the objects (i.e., Bearded vulture, Pyrenean chamois, etc., see Fig. 3).

A probabilistic functional extended $P$ system with active membranes of degree $q \geq 1$ taking $T$ time units, $\Pi=\left(\Gamma, \mu, R, T,\left\{f_{r}: r \in R\right\}\right.$, $\left.M_{0}, \ldots, M_{q-1}\right)$, can be viewed as a skeleton $(\Gamma, \mu, R)$ with the membranes hierarchized by the structure $\mu$ labeled by $0,1, \ldots, q-1 . T$ is a natural number that represents the simulation time of the system. For each rule $r \in R$ and $a, 1 \leq a \leq T, f_{r}(a)$ is a whole number between 0 and 1 , which represents a probabilistic constant associated with rule $r$ at moment $a$. In a generic way, we denote $r: u[v]_{i}^{\alpha f_{r}(a)} \longrightarrow u^{\prime}\left[v^{\prime}\right]_{i}^{\alpha^{\prime}}$.

The tuple of multisets of objects present at any moment in the $q$ regions of the system constitutes the configuration of the system at that moment. The tuple $\left(M_{0}, \ldots, M_{q-1}\right)$ is the initial configuration of $\Pi$. 


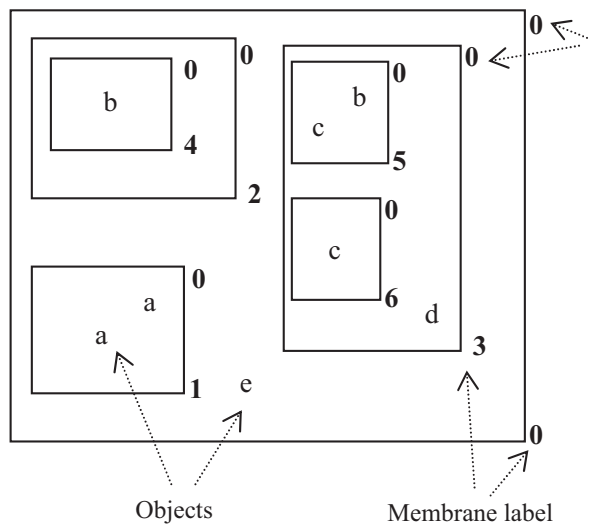

Charge

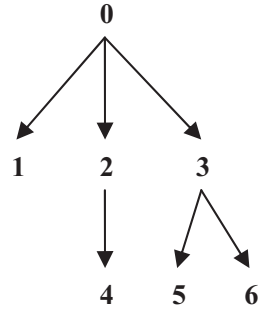

Rules:

$r_{1} \equiv e[d]_{3}^{0} \rightarrow f[a]_{3}^{0}$

$r_{2} \equiv a[]_{6}^{0} \rightarrow[a]_{6}^{+}$

$r_{k} \equiv\left[a^{2}\right]_{1}^{0} \rightarrow[a]_{1}^{0}$

Membranes hierarchized

Fig. 3. A skeleton of an extend P system $\Pi(\Gamma, \mu, R)$ of degree 7 where $\mu=\left[[]_{1}\left[[]_{4}\right]_{2}\left[[]_{5}[]_{6}\right]_{3}\right]_{0}$ and $\Gamma=\{a, b, c, d, e, f\}$.

The P system can pass from one configuration to another by using the rules from $R$ as follows:

- A rule $u[v]_{i}^{\alpha} \stackrel{f_{r}(a)}{\longrightarrow} u^{\prime}\left[v^{\prime}\right]_{i}^{\alpha^{\prime}}$ is applicable to a membrane labeled by $i$, and with $\alpha$ as electrical charge if multiset $u$ is contained in the membrane immediately outside of membrane $i$, it is to say membrane father of membrane $i$, and multiset $v$ is contained in the membrane labeled by $i$ having $\alpha$ as electrical charge. When that rule is applied, multiset $u$ (respectively $v$ ) in the father of membrane $i$ (respectively in membrane $i$ ) is removed from that membrane, and multiset $u^{\prime}$ (respectively $v^{\prime}$ ) is produced in that membrane, changing its electrical charge to $\alpha^{\prime}$.

- $M(\Gamma)$ is the set formed by the multisets of $\Gamma$. If $u, v \in M(\Gamma), \quad i \in\{0, \ldots, q-1\}, \quad \alpha \in\{0,+,-\}$ and $r_{1}, \ldots, r_{z}$ are the rules applicable whose left-hand side is $u[v]_{i}^{\alpha}$ at given moment $a$, then it should be verified that $f_{r_{1}}(a)+\ldots+f_{r_{z}}(a)=1$, and the rules will be applied according to the corresponding probabilities $f_{r_{1}}(a), \ldots, f_{r_{z}}(a)$.

A multienvironment probabilistic functional extended $P$ system with active membranes of degree $(m, q)$ taking $T$ time units

$$
\begin{aligned}
& \left(\Sigma, G, R_{E}, \Gamma, \mu, R, T,\left\{f_{r j}: \quad r \in R_{\Pi}, 1 \leq j \leq m\right\}, M_{i j}:\right. \\
& \quad 0 \leq i \leq q-1,1 \leq j \leq m)
\end{aligned}
$$

can be viewed as a set of $m$ environments $e_{1}, \ldots, e_{m}$ linked by the arcs from the directed graph $G$. Each environment $e_{j}$ contains a probabilistic functional extended $P$ system with active membranes of degree $q, \Pi_{j}=\left(\Gamma, \mu, R, T,\left\{f_{r j}: r \in R_{\Pi}, 1 \leq j \leq m\right\}, M_{i j}: 0 \leq i \leq q-1\right.$, $1 \leq j \leq m)$ each of them with the same skeleton, $\Pi=(\Gamma, \mu, R)$, and such that $M_{0 j}, \ldots, M_{q-1 j}$ describes their initial multisets. $\Sigma$ is an alphabet that represents the objects of $\Gamma$ that can be present in the different environments (Fig. 4).

The communication rule between environments in $R_{E}$ are of the form $r_{e}:(x)_{e_{j}} \stackrel{p_{x, j, k}}{\longrightarrow}(y)_{e_{k}}$, and for each $x \in \Sigma, 1 \leq j \leq m, 1 \leq a \leq T$, it verifies $\sum_{k=1}^{m} p_{x, j, k}(a)=1$. When a rule of this type is applied the object $x$ moves from environment $e_{j}$ to environment $e_{k}$ converted into $y$, according to the probability $p_{j, k}$.

We assume that a global clock exists, marking the time for the whole system (for its compartments), that is, all membranes and the application of all rules are synchronized. In the P systems, a configuration consists of multisets of objects present in the $m$ environments and at each of the regions of the P systems located in the environment.

The $P$ system can pass from one configuration to another by using the rules from $R=R_{E} \cup \cup_{j=1}^{m} R_{\Pi_{j}}$ as follows: at each transition step, the rules to be applied are selected according to the probabilities assigned to them, and all applicable rules are simultaneously applied and all occurrences of the left-hand side of the rules are consumed, as usual.

\subsubsection{The model}

Firstly, we graphically present the problem to be modeled by means of networks that are descriptors of ecological systems that can show the composition of numerous elements and the interactions among them (Bascompte, 2009). The network approach provides a powerful representation of the ecological interac-

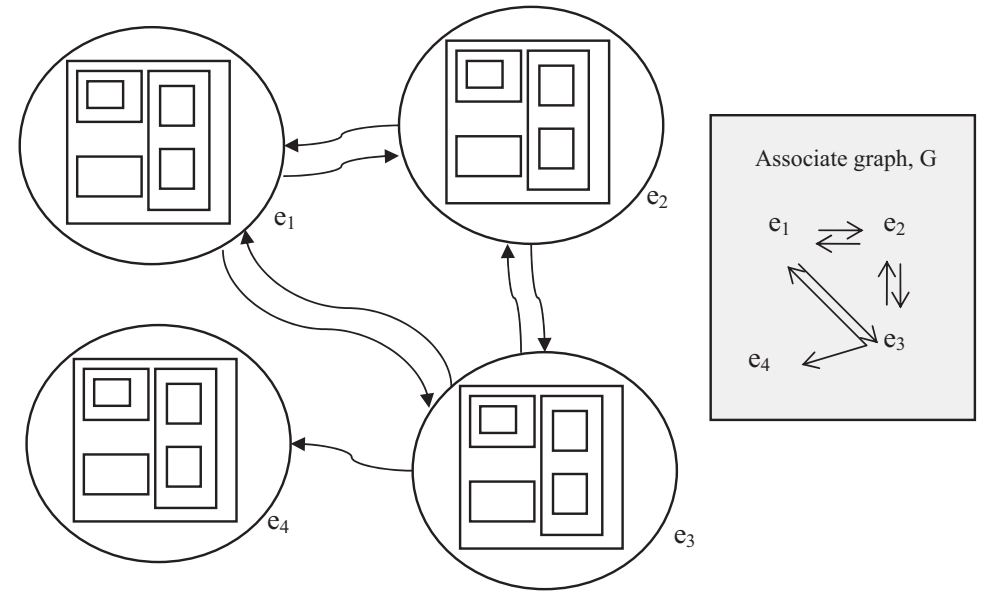

Fig. 4. Multienvironment probabilistic functional extended P system with active membranes of degree $(4,7)$ (four environments and seven membranes). 

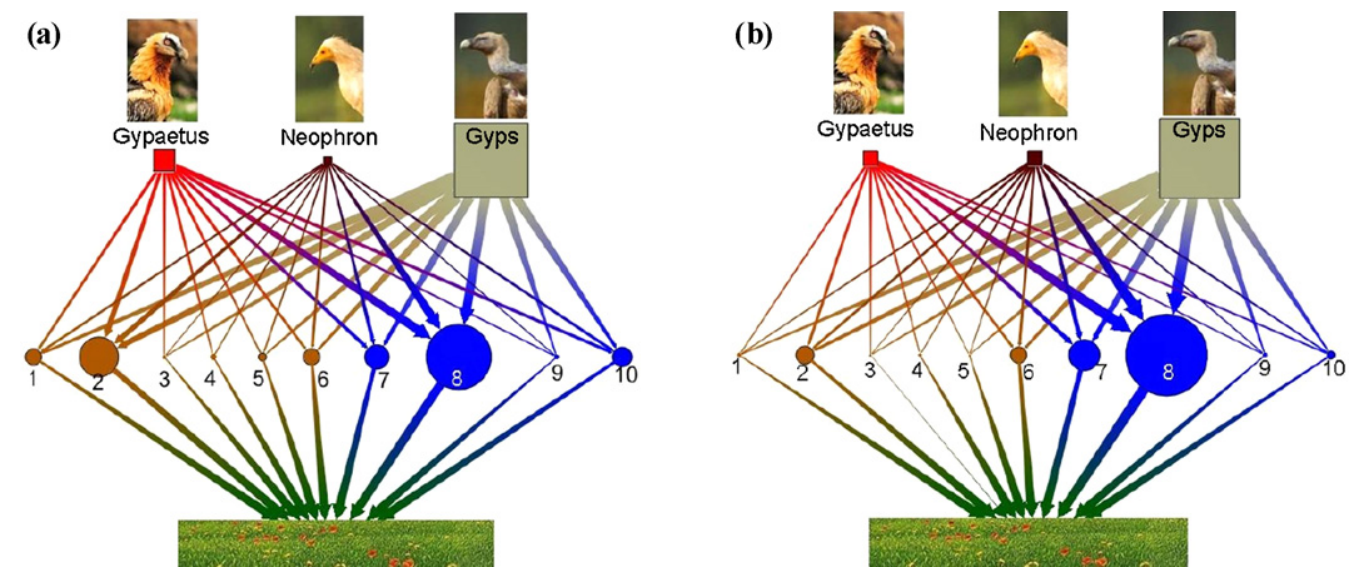

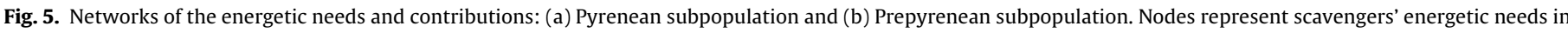

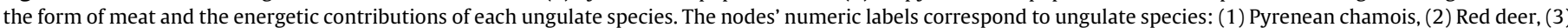

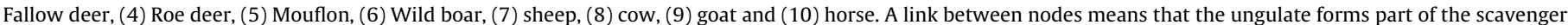
feeding. The thickness of the link denotes the energetic contributions and needs of all the species as a whole and is expressed in percentages.

tions among species and highlights their global interdependence (Ulanowicz, 2004; Bascompte, 2009; Miehls et al., 2009). In this sense, the strength of the interaction among the three species of avian scavengers (predators) on the ungulate community (preys) is quantified for each subpopulation, and is measured as the biomass provided by prey species and the energetic requirements of these three avian scavenger species that are expressed as megacalories per year according to the population size. We consider the vegetable biomass on which wild and domestic ungulates depend as not being a limiting factor but exceeding the needs (energetic requirements) of these species (García, 2008). Subsequently, the model presented associates a rule quantifying the interaction to each of the relations shown in the networks (Fig. 5). In the networks, the energetic needs in the form of meat of the three species of avian scavengers studied inhabiting the Pyrenees (Fig. 5a) and the Prepyrenees (Fig. 5b), are shown by means of nodes as is the proportion of biomass made up by wild and domestic ungulates.

The model proposed must consider: (a) the population dynamics of the nine wild species (three avian scavengers and six ungulates) and four domestic species, (b) the interactions among the 13 species, (c) the presence of two zones in the study area, (d) the communication protocol between the two areas and (e) the ecosystem maximum load capacity for each of the areas.

In order to model the ecosystem, we use a multienvironment probabilistic functional extended $P$ system with active membranes of degree (2,2) (two membranes and two environments) taking $T$ time units (simulation years).

$$
\begin{aligned}
& \left(\Sigma, G, R_{E}, \Gamma, \mu, R, T\left\{f_{r j}: \quad r \in R_{\Pi}, 1 \leq j \leq 2\right\}, M_{i j}:\right. \\
& \quad 0 \leq i \leq 1,1 \leq j \leq 2)
\end{aligned}
$$

The skeleton consists of the working alphabet, $\Gamma$, formed by all objects that belong to the initial configuration and the objects that appear in the evolution of the P system (all of them appear in Appendix A). The membrane structure is formed by the skin membrane labeled 0 and an in membrane labeled 1, both having neutral charge. The set of rules are shown in the appendix; there are 49 types of rules.

The probabilistic functional extended $P$ system $\Pi=(\Gamma, \mu, R, T$, $\left.\left\{f_{r}: r \in R_{\Pi}\right\}, M_{0}, M_{1}\right)$ is defined as follows: for each $r, f_{r}$ is a constant function, the initial configuration is $M_{0}=\left\{X^{q_{i, j}}, d_{i}\right\}$ and $M_{1}=\left\{R_{0}\right.$, $F_{0}$. The objects $X_{i, j, 1}$ are associated with one animal belonging to the species $i, j$ years old in the instance 1 and $q_{i, j}$ is the amount of objects $X_{i, j, 1}$, we use the object $d_{i}$ for a control of the maximum load of the animals of species $i . F_{0}$ is used for generating external contributions of different kinds of food, and finally, object $R_{0}$ allows us to synchronize the P system.

The alphabet that can be present in the environment is $\Sigma=$ $\left\{Z_{i, j, s}, Z_{i, j, s}^{\prime}\right\}$ and the relationships in graph $G$ are of each environment to itself and to other environments.

The algorithmic scheme of the model is structured following a series of modules which are run sequentially corresponding to the passing of 1 year in the ecosystem (Fig. 6). Except for reproduction, all the processes are continuous and annual. In the model, processes are discretized in Cardona et al. (2009), which verified that the order in which modules are applied does not affect the final result obtained from the model.

Some ungulates (Red deer and all domestic ungulates) of the ecosystem have been classified into two different groups according to their management. Specifically, in the case of the Red deer, sexes have been separated because mortality in males is higher than in females due to hunting activities. Thus, the total number of groups of animals considered is 18 . The spatio-temporal distribution of domestic animals has been determined bearing in mind that some animals spend the entire year in the study area whereas others spend variable periods of time there (e.g., summer transhumance). Regarding transhumance, sheep, cows and horses are in mountain passes for 6 months of the year, making use of summer grazing (from mid May to mid October depending on the climate; Roigé, 1995). Data about transhumance were taken from the information provided by the Departament d'Agricultura $i$ Ramaderia of the autonomous government of Catalonia.

The model used is based on objects that evolve by means of a series of rules among which those associated with the interactions shown in the Networks are found (see Fig. 5). Objects $X_{i, j, y}, Y_{i, j, y}, Z_{i, j, y}, Z_{i, j, y}^{\prime}$ and $W_{i, j, y}$ represent the same animal at its different stages throughout the module series. Within the objects, the first index codifies the group to which the animal belongs, the second one codifies its age and the third one the simulation year. The number of years $(T)$ to be simulated is an input of the model.

\subsubsection{Reproduction module}

At the initial instance, an object of type $X$ is associated with each animal. When rules from the reproduction module are applied to objects $X$, they evolve to objects of type $Y$. Objects $X_{i, j}$, associated with females that reproduce when they reach fertility also generate objects $Y_{i, 0, y}$ associated to newborn animals. In this module, objects associated with the amount of food produced by the ecosystem itself (grazing or external contributions of 


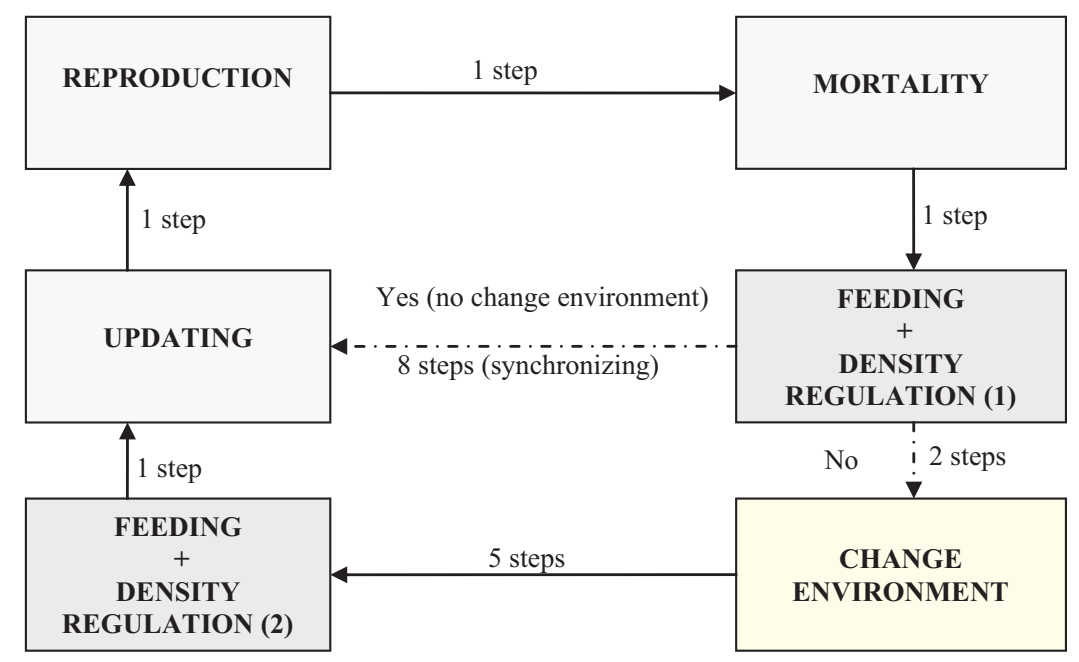

Fig. 6. Modules that form the model.

meat and bones by man) are also generated. This module takes one simulation step. Breeding parameters of each species were obtained from the literature and unpublished data (see Donázar, 1993; Grande, 2006; Margalida et al., 2003; Oro et al., 2008; Le Gouar et al., 2008, see Table 1, Appendix A).

\subsubsection{Mortality module}

Hunting, in the case of wild animals, and mortality due to both natural causes and human management, in the case of domestic animals, provide the animal biomass on which avian scavengers feed. Their survival has been estimated by using bibliographical references (Montserrat and Villar, 2007; Blasco et al., 1992; Casasús et al., 1999; Margalida et al., 2009) as well as by means of personal surveys (authors unpubl. data). Moreover, a percentage of dead animals that may be accessible to scavengers was estimated. The input of this module is formed by objects of the type $Y_{i, j, y}$, which do not evolve but rather move to another membrane and generate a new object D for each animal that does not die. Objects associated with animals that die evolve to objects associated with meat $(C, M)$ and bones $(H, B)$. This module takes one simulation step.

\subsubsection{Feeding and density regulation module (1)}

Annual energetic requirements (expressed as calories or megacalories) as well as the maximum load capacity in the area under study have been estimated for all the species. In this sense, there is no overexploitation of mountain grazing and cattle raising loads have decreased in recent years such that the vegetable biomass available is not a limiting factor (García, 2008). Regarding the avian scavenger community, its population has increased in the last 20 years and maximum values have been estimated according to the unoccupied space as well as to the maximum density that each of these species can achieve (see Table 1, Appendix A). Whether or not the maximum load capacity of the ecosystem for each species has been reached is determined by using objects D previously generated for each surviving animal. Furthermore, objects $Y$ evolve to objects $Z$ to begin the feeding process. In the second step of this module, objects $Z$ evolve to objects $W$ if there is enough physical space and food.

\subsubsection{Change environment module}

When one of the subareas reaches its maximum load capacity, it has been considered that any of the species modeled can move to another subarea according to its ecological requirements. This module will apply if there is some object $Z$ that has not evolved in the previous step; that is, if the resources have been insufficient for all of the animals. In this case, the model simulates animals' movements to find the necessary resources for their survival. Objects $Z$ go out to the environment by taking two simulation steps and subsequently move to another environment by evolving to objects $Z^{\prime}$. Next, in two more steps, they enter the inner membrane of the $P$ system where the objects associated with the possible unused resources are found. This module takes five simulation steps.

\subsubsection{Feeding and density regulation module (2)}

This module will apply if there are enough resources for animals coming from other areas. In such a case, objects $Z^{\prime}$ evolve to objects of type $W$ in one simulation step.

\subsubsection{Updating module}

This module will apply after eight simulation steps; that is, after the running of the third module. After running one cycle within the module series, the initial configuration must be re-established such that a new year (cycle) begins. Objects associated with the surviving animals, $W_{i, j, y}$, evolve to objects $X_{i, j+1, y+1}$. The remaining food is removed and the objects associated with those animals which have not survived evolve to objects representing food. This module takes one simulation step.

In summary, the running of one cycle in the module succession takes 11 simulation steps and represents the passing of a 1-year period in the ecosystem. After the running of the cycle, the P system returns the number of living animals of each species as well as the resources in the form of meat that each has contributed.

\section{Results}

For execution of the model, MeCoSim software (free software under licence), developed by members of the Natural Computation Group at the University of Sevilla (GNU GPL; http://www.plingua.org), has been used.

The population trend of the three scavenger species and the six wild ungulates obtained by the model with respect to data obtained by direct censuses from 1994 to 2008 is shown in Figs. 7 and 8 . In order to check and validate the model, only initial (1994) and final (2008) data were available for the six wild ungulates whereas inter-annual censuses were available for avian scavengers. The population trend of the species present in the ecosystem throughout the period under study has been obtained by running the simulator 100 times for 14 years with the same input data. The simulator executions have allowed us to estimate the population confidence intervals of the different species. The ecosystem mod- 
Table 1

Values of parameters used in the model for each species ( $\mathrm{F}=$ female, $\mathrm{M}=$ male, $\mathrm{A}=$ spend the entire year in the mountain, $\mathrm{P}=$ spend part of the year in the mountain),

\begin{tabular}{|c|c|c|c|c|c|c|c|c|c|c|c|c|c|c|c|c|c|c|c|c|c|c|c|}
\hline & $g_{1} \mid$ & $g_{2}$ & $g_{3} \mid$ & $g_{4} \mid$ & $g_{5}$ & $g_{6} \mid$ & $g_{7} \mid$ & $k_{1}$ & $k_{2}$ & $k_{3}$ & $m_{1} \mid$ & $m_{2}$ & $m_{3}$ & $m_{4}$ & $f_{1}$ & $f_{2}$ & $f_{3}$ & $f_{4}$ & $f_{5}$ & $f_{6}$ & $f_{7}$ & $f_{8}$ & $f_{9}$ \\
\hline Gypaetus barbatus & 1 & 1 & 1 & 6 & 20 & 21 & 0 & 0.65 & 0.35 & 1 & 0.06 & 0.08 & 0 & 1 & 0 & 0 & 0 & 0 & 135 & 0 & 45 & 0 & 0 \\
\hline Neophron percnopterus & 1 & 0.5 & 1 & 5 & 24 & 25 & 1 & 0.80 & 0.57 & 1 & 0.28 & 0.08 & 0 & 1 & 0 & 0 & 0 & 0 & 0 & 0 & 100 & 0 & 0 \\
\hline Gyps fulvus & 1 & 1 & 1 & 5 & 24 & 25 & 0 & 0.75 & 0.56 & 1 & 0.06 & 0.07 & 0 & 1 & 0 & 0 & 0 & 0 & 0 & 0 & 230 & 0 & 0 \\
\hline Rupicapra pyrenaica & 1 & 1 & 1 & 2 & 18 & 18 & 0 & 0.55 & 0.75 & 1 & 0.6 & 0.06 & 0 & 1 & 3 & 4 & 6 & 24 & 0 & 0 & 0 & 0.5 & 0.5 \\
\hline Cervus elaphus (Female) & 1 & 1 & 1 & 2 & 17 & 17 & 0 & 1 & 0.75 & 1 & 0.34 & 0.06 & 0 & 1 & 7 & 13 & 15 & 60 & 0 & 0 & 0 & 0.6 & 0.6 \\
\hline Cervus elaphus (Male) & 1 & 1 & 1 & 2 & 20 & 20 & 0 & 0 & 0 & 0 & 0.34 & 0.06 & 0 & 1 & 12 & 15 & 24 & 96 & 0 & 0 & 0 & 0.6 & 0.6 \\
\hline Dama dama & 1 & 1 & 1 & 2 & 12 & 12 & 0 & 0.75 & 0.55 & 1 & 0.5 & 0.06 & 0 & 1 & 1 & 14 & 2 & 37 & 0 & 0 & 0 & 0.25 & 0.25 \\
\hline Capreolus capreolus & 1 & 1 & 1 & 1 & 10 & 10 & 0 & 0.67 & 1 & 1 & 0.58 & 0.06 & 0 & 1 & 1 & 4 & 1 & 19 & 0 & 0 & 0 & 0.25 & 0.25 \\
\hline Ovis orientalis & 1 & 1 & 1 & 2 & 12 & 12 & 0 & 0.5 & 0.9 & 2 & 0.6 & 0.06 & 0 & 1 & 3 & 4 & 6 & 22 & 0 & 0 & 0 & 0.6 & 0.6 \\
\hline Sus scrofa & 1 & 1 & 1 & 1 & 4 & 6 & 0 & 0.5 & 0.55 & 4 & 0.14 & 0.1 & 0 & 1 & 4 & 6 & 12 & 60 & 0 & 0 & 0 & 0.25 & 0.25 \\
\hline Ovis aries (Adult) & 0 & 1 & 1 & 2 & 8 & 8 & 0 & 0.96 & 0.75 & 1 & 0.15 & 0.03 & 0 & 0 & 3 & 4 & 7 & 38 & 0 & 0 & 0 & 0.7 & 0.7 \\
\hline Ovis aries (Young) & 0 & 0.5 & 1 & 2 & 8 & 8 & 0 & 0.96 & 0.75 & 1 & 0.15 & 0.03 & 0 & 0 & 3 & 4 & 7 & 38 & 0 & 0 & 0 & 0.7 & 0.7 \\
\hline Bos taurus (Adult) & 0 & 1 & 2 & 2 & 9 & 14 & 0 & 0.9 & 0.9 & 1 & 0.057 & 0.045 & 0 & 0 & 10 & 60 & 6 & 518 & 0 & 0 & 0 & 0.6 & 0.6 \\
\hline Bos taurus (Young) & 0 & 0.4 & 2 & 2 & 9 & 14 & 0 & 0.9 & 0.9 & 1 & 0.057 & 0.045 & 0 & 0 & 10 & 60 & 6 & 518 & 0 & 0 & 0 & 0.6 & 0.6 \\
\hline Capra hircus (Adult) & 0 & 1 & 1 & 2 & 8 & 8 & 0 & 0.97 & 0.9 & 1 & 0.12 & 0.015 & 0 & 0 & 3 & 4 & 9 & 37 & 0 & 0 & 0 & 0.6 & 0.6 \\
\hline Capra hircus (Young) & 0 & 0.5 & 1 & 2 & 8 & 8 & 0 & 0.97 & 0.9 & 1 & 0.12 & 0.015 & 0 & 0 & 3 & 4 & 9 & 37 & 0 & 0 & 0 & 0.6 & 0.6 \\
\hline Equus caballus (Adult) & 0 & 1 & 3 & 3 & 9 & 20 & 0 & 0.97 & 0.9 & 1 & 0.034 & 0.0142 & 0 & 0 & 10 & 60 & 9 & 891 & 0 & 0 & 0 & 0.8 & 0.8 \\
\hline Equus caballus (Young) & 0 & 0.55 & 3 & 3 & 9 & 20 & 0 & 0.97 & 0.9 & 1 & 0.034 & 0.0142 & 0 & 0 & 10 & 60 & 9 & 891 & 0 & 0 & 0 & 0.8 & 0.8 \\
\hline
\end{tabular}

$\mathrm{g}_{1}: 1$ wild animal and 0 domestic animals.

$g_{2}$ : proportion of time that animals remain in the mountains during the year.

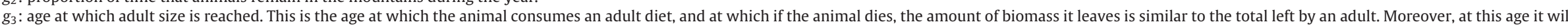
have surpassed the critical early phase during which the mortality rate is high.

$g_{4}$ : age at which fertility begins.

$g_{6}$ : average life expectancy in the ecosystem.

$g_{7}: 1$ if an important proportion of the diet of the species can be based on other small species and 0 for the remainder.

$k_{1}$ : proportion of females in the population (per one).

$k_{2}$ : fertility ratio (proportion of fertile females that reproduce).

$k_{3}$ : number of descendants for fertile females that reproduce.

$m_{1}$ : natural mortality ratio in first years, age $<\mathrm{g}_{4}$ (per one).

$m_{2}$ : mortality ratio in adult animals, age $>g_{4}$ (per one).

$m_{3}$ : percentage of domestic animals removed from non-stabilized populations at early ages.

$m_{4}$ : is equal to 1 if the animal dies at the age of $g_{6}$ and is not removed, and is equal to 0 if the animal does not die at the age of $g_{6}$ but is removed from the ecosystem.

$f_{1}$ : amount of bones from young animals, age $<g_{4}$

$f_{2}$ : amount of meat from young animals, age $<g_{4}$.

$f_{3}$ : amount of bones from adult animals, age $<g_{4}$.

$f_{4}$ : amount of meat from adult animals, age $<g_{4}$

$f_{5}$ : amount of bones necessary per year and animal $(\mathrm{kg})$

$f_{6}$ : amount of grass necessary per year and animal $(\mathrm{kg})$.

$f_{7}$ : amount of meat necessary per year and animal $(\mathrm{kg})$.

$f_{8}$ : Percentage of useful bones.

$f_{9}$ : Percentage of useful meat. 
eled in this work is made up of 13 species totaling 18 animal types. The model shows the population trend obtained by equally dividing the evolutions of each animal. The initial population is composed of 385.422 individuals and the computation time (on a personal computer) for 100 repetitions, corresponding to 14 simulation years each, was $30 \mathrm{~min}$.

The comparison between the real population tendency, estimated by means of censuses carried out, and that obtained by the simulator is shown in Fig. 7. The adjustment of the population trend shown for the three avian scavenger species with respect to the data obtained by the simulator shows that the model functions properly.

With respect to the results obtained for wild ungulates (Fig. 8), it is observed that in simulation year 10 , Roe deer reaches its maximum load capacity in the Prepyrenean area (zone 2) and some of the animals move to the Pyrenean area, causing in the latter an important population increase in simulation year 11 . Thus, the model is able to show the dispersive capacity of some species. A similar situation is observed for Red deer in simulation year 10 and Pyrenean chamois in simulation year 9. Note that as simulations continue beyond the initial year of simulation the confidence interval increases. It is a random model such that results are spread horizontally.

\section{Discussion}

The model presented based on P Systems correctly simulates the population dynamics in the period of time analyzed. Our model considers the population dynamics and the simultaneous interaction among the 18 animal types. In Cardona et al. (2009) we described a model based on P systems (the ecosystem modeled is composed by one avian scavenger species and five prey species) that considers neither the ecosystem maximum load capacity nor the appearance of density-dependent regulatory phenomena in the species. In Cardona et al. (2010a), a new model was presented that overcame some limitations of the previous model by widening the number of species (three avian scavenger species and ten prey species) including some specific characteristics of the species forming the ecosystem. Nonetheless, this model considered the ecosystem to be a closed entity, that is, when the necessary resources for a species to survive (space, feeding,...) are insufficient, the animal dies without considering the possibility that the animals move to some other ecosystem. In this study, we improve the model of Cardona et al. (2010a) considering the heterogeneity of the landscape and the possibility of spatial movements of the species when food resources are enough to cover the energetic requirements of the species.

Models based on individuals such as that presented in this study are generally more flexible and enable the consideration of the heterogeneity of the population and the environment. Our model is composed of modules that are applied sequentially. In our model the advantages with respect to other models are (1) it is not necessary to divide the problem to be analyzed (Fulton et al., 2003, 2004; Lawrie and Hearne, 2008); (2) the number of inter- and intraspecific interactions among individuals is not limited (Mullon et al., 2008); (3) it is able to capture the randomness inherent to the processes; and (4) in the function of ecosystem dynamics, it is able to update the parameters in a functional way, or in other words, to readjust (Cardona et al., 2010a).

Another advantage in the application of our model is that it is easily programmable and is fast compared to the time of computation for a deterministic model with similar characteristics involving $9 \mathrm{~h}$ in one simulation year with 240,000 individuals (Morales et al., 2006).

Among the specialized programs that enable the study of species viability (e.g., Gapps, Inmat, Ramas, Vortex) the most widely used
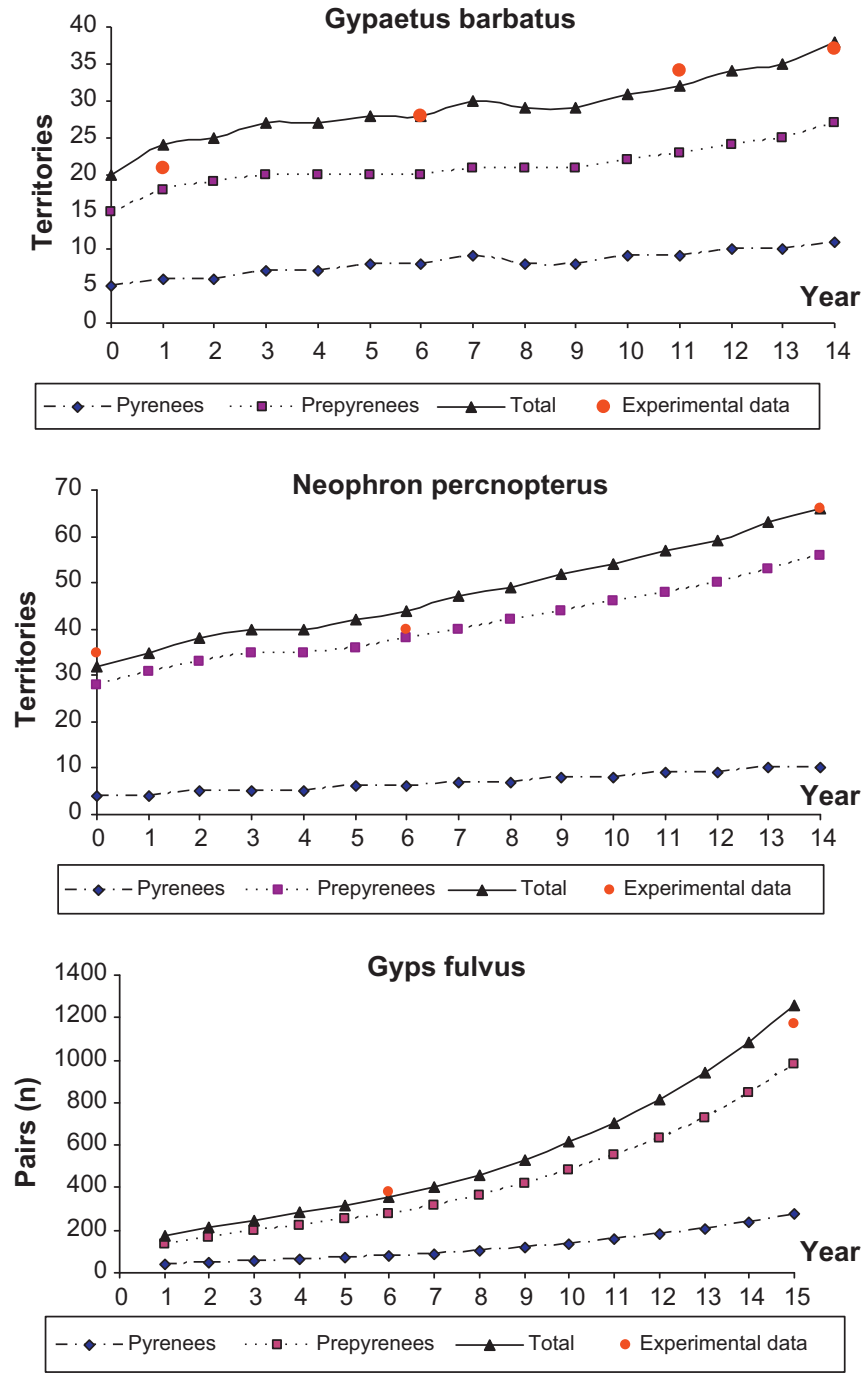

Fig. 7. Comparison of the results obtained by the simulator (lines) with the data obtained experimentally (dots). Unbroken line represents the whole population whereas broken lines represent the results obtained in both subpopulations, Pyrenees: circles; Prepyrenees: squares.

is Vortex, which considers a higher number of factors. A comparative study showed that the results obtained from the diverse programs were different if the models were not normalized (Brook et al., 1999). Like the model presented in this paper, Vortex enables the modeling of different populations and considers fertility ratios, male-female relationships, number of descendants and interactions among different populations. Nonetheless, Vortex considers the possibility that natural disasters may take place whereas the model presented here can only consider such a possibility by including a new module. Finally, Vortex can simultaneously model different populations that interact with each other although they must correspond to the same species. The model used in this work enables the study of the dynamics of different populations whether or not they are composed of the same species, even allowing interaction and competition among them. In addition, the availability of energetic resources that are essential for the dynamics of populations that are interrelated as well as spatio-temporal regulation are considered.

The flexibility of our model enables the increase in the number of species without having to modify the model but simply by adding the new information on the biological parameters of the new species to be included to the simulator or modifying the infor- 

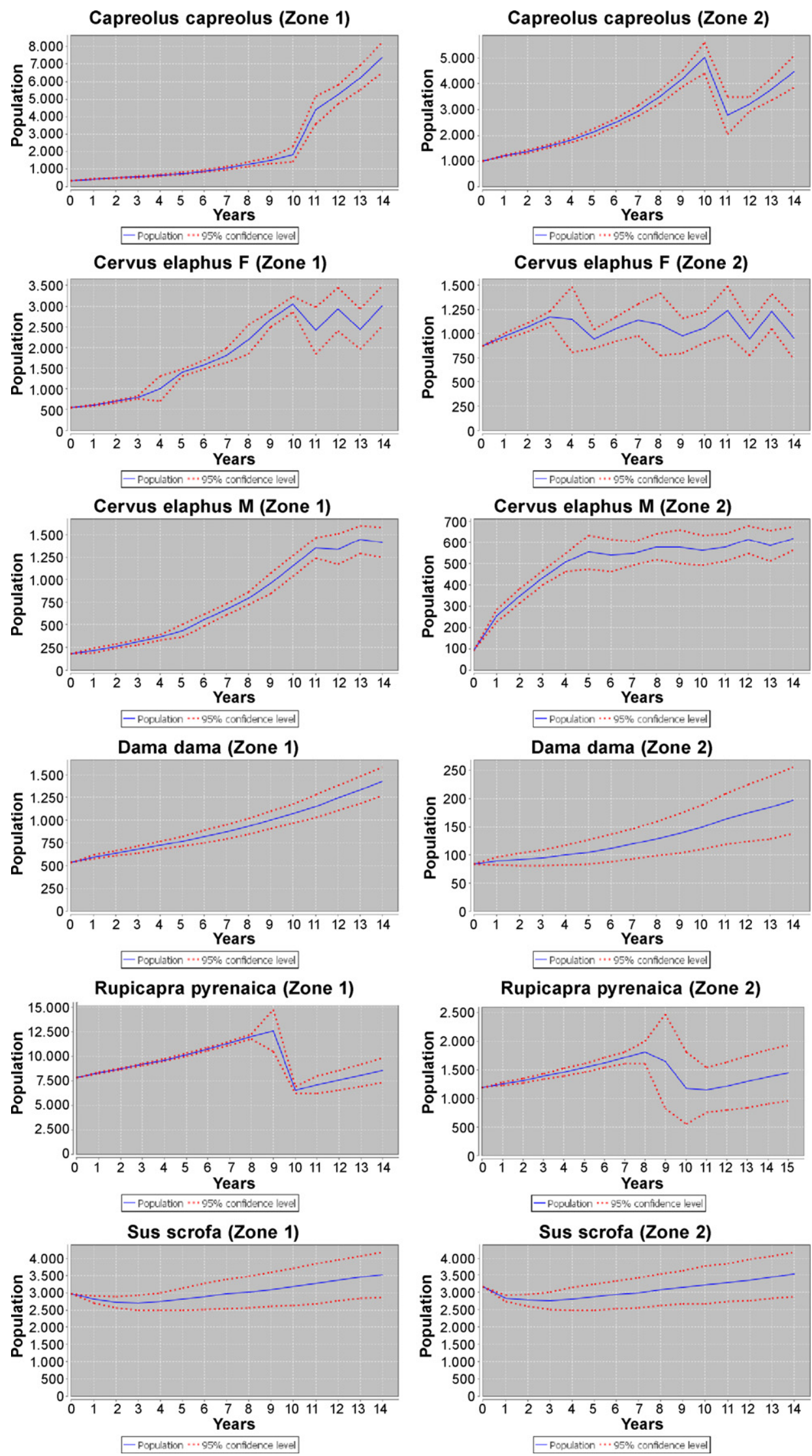

Fig. 8. Results provided by the simulator. The starting point is the population of every species in the year 1994. Zone 1: Pyrenees, Zone 2: Prepyrenees. 
mation available. It is important to highlight that the input data and the data the model works with are obtained directly in the field, which allow us to verify immediately whether or not the input data can be considered correct. It also enables virtual experimentation on population dynamics under different conditions as well as the validation of its usefulness as a simulation tool.

A dynamic tool enabling examination of different scenarios and the evolution of the ecosystem in each case has been presented. The capacity of an objective and robust prediction tool to forecast population tendencies and the future situation of the species involved in the analysis is essential for decision-making by managers and policy-makers. For example, understanding the necessity of supplementary feeding points with respect to the trophic availability or the sanitary legislation regulations (see Donázar et al., 2009; Margalida et al., 2010) can constitute an important tool for the optimization of management action for scavenger species conservation. Given the robustness of the model, it may be possible to model future scenarios with different trophic resource availability to simulate population dynamics. This could allow managers to accurately assess the necessity of increasing food availability (i.e., through the establishment of supplementary feeding sites or the management of wild ungulate populations), the areas with less food availability, or those species on which food increases should be focused.

In short, $\mathrm{P}$ systems present important advantages with respect to traditional models based on differential equations, such as a high computational potential, modularity and ability to work in parallel.

\section{Acknowledgements}

We thank Federic Fillat, Ricardo García-González, Llorenç Ricou, Jordi Ruiz-Olmo, Diego García, Toni Batet, Ivan Afonso and Jordi Canut for data provided. Jordi Bascompte and three anonymous referees reviewed this manuscript.

\section{Appendix A.}

\section{A.1. Description of the model based on P Systems for an ecosystem occupied by three avian scavengers}

The ecosystem modeling is composed of two environments (Pyrenees and Prepyrenees) and 18 types of animals. The number of animals per each species and environment as well as the number of years to be simulated are the input of the model.

The output is formed by the number of animals of each species according to their age, the number of simulation years and the biomass in the form of megacalories that every species provides throughout the years simulated.

In order to model this ecosystem we use a multienvironment probabilistic functional extended $P$ system with active membranes of degree (2,2) (two membranes and two environments) taking $T$ time units (simulation years).

$$
\begin{aligned}
& \left(\Sigma, G, R_{E}, \Gamma, \mu, R, T\left\{f_{r j}: \quad r \in R_{\Pi}, 1 \leq j \leq 2\right\}, M_{i j}:\right. \\
& \quad 0 \leq i \leq 1,1 \leq j \leq 2)
\end{aligned}
$$

The polarization of the membranes are used to show environmental changes (i.e., timing of the year).

1. The membrane structure is $\mu=\left[[]_{1}\right]_{0}$.

The initial configuration is $M_{0}=\left\{X^{q_{i, j}}, d_{i}\right\}$ and $M_{1}=\left\{R_{0}, F_{0}\right\}$ (see Fig. 9). For each animal there exists an object $X_{i, j, 1}, q_{i, j}$ is the multiplicity of object $X_{i, j, 1}$ and thus the number of animals of species $i$ and age $j$. We use object $d_{i}$ to control the maximum load capacity for the animals of species $i . F_{0}$ is used for generating external contributions of different kinds of food, and finally object $R_{0}$ allows us to synchronize the P system.

2. The working alphabet of the P system is

$$
\begin{aligned}
& \Gamma=\left\{X_{i, j, y}, Y_{i, j, y}, Z_{i, j, y}, Z_{i, j, y}^{\prime}, W_{i, j, y}: 1 \leq i \leq n, 0 \leq j \leq g_{i, 6}, 1 \leq y \leq T\right\} \cup \\
& \left\{B, M, S, G, F_{0}, F, b\right\} \cup\left\{R_{i}: \quad 0 \leq i \leq 8 \stackrel{\leftrightarrow}{\longrightarrow} \cup\left\{H_{i}, C_{i}, D_{i}, d_{i}, a_{i}, e_{i}: \quad 1 \leq i \leq n\right\}\right.
\end{aligned}
$$

Objects $X_{i, j, y}, Y_{i, j, y}, Z_{i, j, y}, Z_{i, j, y}^{\prime}$ and $W_{i, j, y}$ represent the same animal but in different states. Index $i$ is associated with the type of animal, index $j$ is associated with the age (and $g_{i, 6}$ is the average life expectancy) and $y$ is the simulation year. Objects $B$ and $H$ represent bones, $C_{i}$ and $M_{i}$ represent meat corresponding to the species $i, S$ represents meat from small animals and $G$ is the amount of grass available for consumption in the ecosystem, $F_{0}$ and $F$ are used to generate external contributions. $D_{i}$ is an object used to count the existing animals of species $i$. If a species overcomes the maximum density values, it will be regulated. Objects $d_{i}, a_{i}$ and $e_{i}$ allow us to control the maximum number of animals per species in the ecosystem. When a regulation takes place, object $a_{i}$ allows us to eliminate the number of animals of species $i$ that exceeds the maximum density. $b$ is an object used to change the charge of the membrane. At the end, object $R_{i}$ is a counter that allows the synchronization of the P system.

3. Environment alphabet is $\Sigma=\left\{Z_{i, j, y}, Z_{i, j, y}^{\prime}: \quad 1 \leq i \leq n, \quad 1 \leq j \leq\right.$ $\left.g_{i, 6}, \quad 1 \leq y \leq T\right\}$

Objects $Z_{i, j, y}$ and $Z_{i, j, y}^{\prime}$ are associated with animals.

4. Rules $R$ and $R_{E}$ of the model.

The definition of parameters that appear in the rules are:

$g_{1}: 1$ wild animal and 0 domestic animals.

$g_{2}$ : proportion of time that animals remain in the mountains during the year.

$g_{3}$ : age at which adult size is reached. This is the age at which the animal consumes an adult diet, and at which if the animal dies, the amount of biomass it leaves is similar to the total left by an adult. Moreover, at this age it will have surpassed the critical early phase during which the mortality rate is high.

$g_{4}$ : age at which fertility begins.

$g_{5}$ : age at which fertility ends.

$g_{6}$ : average life expectancy in the ecosystem.

$g_{7}: 1$ if an important proportion of the diet of the species can be based on other small species and 0 for the remainder.

$k_{1}$ : proportion of females in the population (per one).

$k_{2}$ : fertility ratio (proportion of fertile females that reproduce).
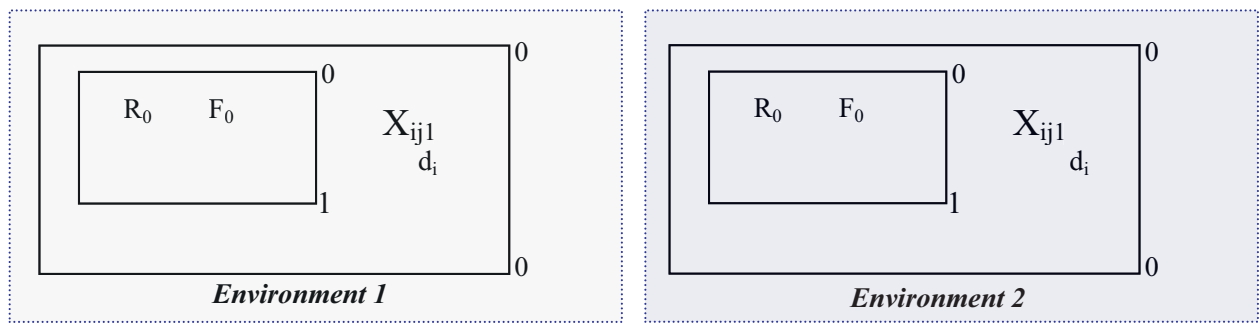

Fig. 9. Model based on P systems. Initial configuration. Environment 1 (Pyrenees); Environment 2 (Prepyrenees). The multiplicity of the object is omitted. 
$k_{3}$ : number of descendants for fertile females that reproduce. $m_{1}$ : natural mortality ratio in first years, age $<g_{4}$ (per one).

$m_{2}$ : mortality ratio in adult animals, age $\geq g_{4}$ (per one).

$m_{3}$ : percentage of domestic animals removed from nonstabilized populations at early ages.

$m_{4}$ : is equal to 1 if the animal dies at the age of $g_{6}$ and is not removed, and is equal to 0 if the animal does not die at the age of $g_{6}$ but is removed from the ecosystem.

$f_{1}$ : amount of bones from young animals, age $<g_{4}$.

$f_{2}$ : amount of meat from young animals, age $<g_{4}$.

$f_{3}$ : amount of bones from adult animals, age $<g_{4}$.

$f_{4}$ : amount of meat from adult animals, age $<g_{4}$.

$f_{5}$ : amount of bones necessary per year and animal $(\mathrm{kg})$.

$f_{6}$ : amount of grass necessary per year and animal $(\mathrm{kg})$.

$f_{7}$ : amount of meat necessary per year and animal $(\mathrm{kg})$.

$f_{8}$ : Percentage of useful bones.

$f_{9}$ : Percentage of useful meat.

$d_{k, i, 1}$ : maximum density of species $i$ in the ecosystem $k$.

$d_{k, i, 2}$ : number of animals of species i that survive after they reach the maximum density of the ecosystem $k$.

$p_{i, k, v}$ : probability that species $i$ will move from environment $k$ to environment $v$ when there is a lack of resources.

The values of the parameters for each species are shown in Tables 1 and 2 .

- Reproduction module

Rule $r_{0}$ allows the generation of objects associated with meat, bones and grass corresponding to the amount of these resources provided externally or by the ecosystem itself (i.e., additional grass or meat provided by small birds, mammals or reptiles present in the ecosystem).

$r_{0} \equiv\left(\left[F_{0} \rightarrow B^{\alpha(k)} M^{\beta(k)} S^{\lambda(k)} G^{\gamma(k)} F\right]_{1}^{0}\right)_{e_{k}}, 1 \leq k \leq 2$.

$\alpha(k), \beta(k), \lambda(k)$ and $\gamma(k)$ are the amount of bones, meat, meat of small animals and grass provided externally.

Objects $a_{i}$ are generated to verify the maximum charge capacity per species. Object $e_{i}$ permits randomness in the number of animals of a species when the ecosystem maximum charge capacity is reached.

$$
r_{1} \equiv\left(d_{i}[]_{1}^{0} \rightarrow\left[d_{i} a_{i}^{0,9 \cdot d_{k, i, 1}} e_{i}^{0,2 \cdot d_{k, i, 1}}\right]_{1}^{0}\right)_{e_{k}},\left\{\begin{array}{l}
1 \leq k \leq 2 \\
1 \leq i \leq n
\end{array}\right.
$$

Table 2

Probability that the species moves between environments. $p_{i, k, v}$ : probability that species $i$ will move from environment $k$ to environment $v$ when there is a lack of resources.

\begin{tabular}{llllll}
\hline & $i$ & $p\left(i, e_{1}, e_{1}\right)$ & $p\left(i, e_{1}, e_{2}\right)$ & $p\left(i, e_{2}, e_{1}\right)$ & $p\left(i, e_{2}, e_{2}\right)$ \\
\hline Gypaetus barbatus & 1 & 0 & 1 & 0 & 1 \\
Neophron percnopterus & 2 & 0 & 1 & 0 & 1 \\
Gyps fulvus & 3 & 0 & 1 & 0 & 1 \\
Rupicapra pyrenaica & 4 & 0.95 & 0.05 & 0.95 & 0.05 \\
Cervus elaphus (Female) & 5 & 0.8 & 0.2 & 0.8 & 0.2 \\
Cervus elaphus (Male) & 6 & 0.8 & 0.2 & 0.8 & 0.2 \\
Dama dama & 7 & 0.98 & 0.02 & 0.98 & 0.02 \\
Capreolus capreolus & 8 & 0.7 & 0.3 & 0.7 & 0.3 \\
Ovis orientalis & 9 & 0.995 & 0.005 & 0.995 & 0.005 \\
Sus scrofa & 10 & 0.98 & 0.02 & 0.98 & 0.02 \\
Ovis aries (Adult) & 11 & 1 & 0 & 1 & 0 \\
Ovis aries (Young) & 12 & 1 & 0 & 1 & 0 \\
Bos taurus (Adult) & 13 & 1 & 0 & 1 & 0 \\
Bos taurus (Young) & 14 & 1 & 0 & 1 & 0 \\
Capra hircus (Adult) & 15 & 1 & 0 & 1 & 0 \\
Capra hircus (Young) & 16 & 1 & 0 & 1 & 0 \\
Equus caballus (Adult) & 17 & 1 & 0 & 1 & 0 \\
Equus caballus (Young) & 18 & 1 & 0 & 1 & 0 \\
\hline
\end{tabular}

Reproduction rules:

Males that do not reproduce.

$r_{2} \equiv\left[X_{i, j, y} \stackrel{\left(1-k_{i, 1}\right)}{\longrightarrow} Y_{i, j, y}\right]_{0}^{0},\left\{\begin{array}{l}1 \leq i \leq n, \\ g_{i, 4} \leq j<g_{i, 5}, \\ 1 \leq y \leq T .\end{array}\right.$

Females at fertile ages that reproduce.

$r_{3} \equiv\left[X_{i, j, y} \stackrel{k_{i, 1} \cdot k_{i, 2}}{\longrightarrow} Y_{i, j, y} Y_{i, o, y}^{k_{i, 3}}\right]_{0}^{0}, \quad\left\{\begin{array}{l}1 \leq i \leq 4, \\ g_{i, 4} \leq j<g_{i, 5}, \\ 1 \leq y \leq T .\end{array}\right.$

$r_{4} \equiv\left[X_{i, j, y} \stackrel{k_{i, 1} \cdot k_{i, 2}}{\longrightarrow} Y_{i, j, y} Y_{i, o, y}^{k_{i, 3}}\right]_{0}^{0}, \quad\left\{\begin{array}{l}7 \leq i \leq n, \\ g_{i, 4} \leq j<g_{i, 5}, \\ 1 \leq y \leq T .\end{array}\right.$

$r_{5} \equiv\left[X_{5, j, y} \stackrel{0.5 \cdot k_{5,2}}{\longrightarrow} Y_{5, j, y} Y_{5, o, y}^{k_{5,3}}\right]_{0}^{0}, \quad\left\{\begin{array}{l}g_{5,4} \leq j<g_{5,5}, \\ 1 \leq y \leq T .\end{array}\right.$

$r_{6} \equiv\left[X_{5, j, y} \stackrel{0.5 \cdot k_{5}, 2}{\longrightarrow} Y_{5, j, y} Y_{6, o, y}^{k_{5,3}}\right]_{0}^{0}, \quad\left\{\begin{array}{l}g_{5,4} \leq j<g_{5,5}, \\ 1 \leq y \leq T .\end{array}\right.$

The Red deer species has been divided into two groups: $i=5$ are females and $i=6$ are males. This is caused by the fact that a higher mortality rate occurs in males compared to females due to hunting activities.

Females at fertile ages that do not reproduce.

$r_{7} \equiv\left[X_{i, j, y} \stackrel{k_{i, 1} \cdot\left(1-k_{i, 2}\right)}{\longrightarrow} Y_{i, j, y}\right]_{0}^{0}, \quad\left\{\begin{array}{l}1 \leq i \leq n, \\ g_{i, 4} \leq j<g_{i, 5}, \\ 1 \leq y \leq T .\end{array}\right.$

Adult non-fertile males and females

$r_{8} \equiv\left[X_{i, j, y} \rightarrow Y_{i, j, y}\right]_{0}^{0}, \quad\left\{\begin{array}{l}1 \leq i \leq n, \\ g_{i, 5} \leq j \leq g_{i, 6} \\ 1 \leq y \leq T\end{array}\right.$

Non-fertile young animals.

$r_{9} \equiv\left[X_{i, j, y} \rightarrow Y_{i, j, y}\right]_{0}^{0}, \quad\left\{\begin{array}{l}1 \leq i \leq n, \\ 1 \leq j \leq g_{i, 4} \\ 1 \leq y \leq T\end{array}\right.$

After applying the rules prior to the initial configuration, configuration 1 is obtained (see Fig. 10).

- Mortality module

Mortality rules.

Young animals that survive.

$r_{10} \equiv Y_{i, j, y}[]_{1}^{01-m_{i, 1}-m_{i, 3}}\left[Y_{i, j, y} D_{i}\right]_{1}^{+}, \quad\left\{\begin{array}{l}1 \leq i \leq n, \\ 0 \leq j<g_{i, 3}, \\ 1 \leq y \leq T .\end{array}\right.$ 
Young animals that die and leave biomass in the form of meat and bones.

$$
\begin{aligned}
r_{11} & \equiv Y_{i, j, y}[]_{1}^{0} \stackrel{m_{i, 1}}{\longrightarrow}\left[H_{i}^{f_{i, 1}} \cdot g_{i, 2} \cdot f_{i, 8} C_{i}^{f_{i, 2}} \cdot g_{i, 2} \cdot f_{i, 9} B_{i}^{f_{i, 1}} \cdot g_{i, 2} \cdot f_{i, 8} M_{i}^{f_{i, 2}} \cdot g_{i, 2} \cdot f_{i, 9}\right]_{1}^{+}, \\
& \left\{\begin{array}{l}
1 \leq i \leq n, \\
0 \leq j<g_{i, 3}, \\
1 \leq y \leq T .
\end{array}\right.
\end{aligned}
$$

Young animals removed from the ecosystem that do not leave biomass.

$r_{12} \equiv Y_{i, j, y}[]_{1}^{0} \stackrel{m_{i, 3}}{\longrightarrow}[b]_{1}^{+}, \quad\left\{\begin{array}{l}1 \leq i \leq n, \\ 0 \leq j<g_{i, 3}, \\ 1 \leq y \leq T .\end{array}\right.$

Adult animals that survive.

$r_{13} \equiv Y_{i, j, y}[]_{1}^{01-m_{i, 2}}\left[Y_{i, j, y} D_{i}\right]_{1}^{+}, \quad\left\{\begin{array}{l}1 \leq i \leq n, \\ g_{i, 3} \leq j<g_{i, 6} \\ 1 \leq y \leq T .\end{array}\right.$

Adult animals that die and leave biomass.

$$
\begin{aligned}
& r_{14} \equiv Y_{i, j, y}[]_{1}^{0} \stackrel{m_{i, 2}}{\longrightarrow}\left[H_{i}^{f_{i, 3}} \cdot g_{i, 2} \cdot f_{i, 8} C_{i}^{f_{i, 4} \cdot g_{i, 2} \cdot f_{i, 9} B^{f_{i, 3}} \cdot g_{i, 2} \cdot f_{i, 8}} M^{f_{i, 4} \cdot g_{i, 2} \cdot f_{i, 9}}\right]_{1}^{+}, \\
& \left\{\begin{array}{l}
1 \leq i \leq n, \\
g_{i, 3} \leq j<g_{i, 6}, \\
1 \leq y \leq T .
\end{array}\right.
\end{aligned}
$$

Animals that reach average life expectancy and die in the ecosystem leaving biomass

$$
\begin{aligned}
r_{15} & \equiv Y_{i, g_{i, 6}, y}[]_{1}^{0 m_{i, 4}+\left(1-m_{i, 4}\right) \cdot m_{i, 2}}\left[H_{i}^{f_{i, 3} \cdot g_{i, 2} \cdot f_{i, 8}} C_{i}^{f_{i, 4} \cdot g_{i, 2} \cdot f_{i, 9}} B_{B_{i, 3} \cdot g_{i, 2} \cdot f_{i, 8}} M^{f_{i, 4} \cdot g_{i, 2} \cdot f_{i, 9}}\right]_{1}^{+}, \\
& \left\{\begin{array}{l}
1 \leq i \leq n, \\
1 \leq y \leq T .
\end{array}\right.
\end{aligned}
$$

Animals that reach average life expectancy and leave the ecosystem without leaving biomass.

$$
r_{16} \equiv\left[Y_{i, g_{i, 6, y}} \stackrel{\left(1-m_{i, 4}\right) \cdot\left(1-m_{i, 2}\right)}{\longrightarrow} \#\right]_{0}^{0},\left\{\begin{array}{l}
1 \leq i \leq n, \\
1 \leq y \leq T .
\end{array}\right.
$$

Randomness generation of the total amount of animals.

$$
r_{17} \equiv\left[e_{i}\right]_{1}^{0} \stackrel{0.5}{\longrightarrow}\left[a_{i}\right]_{1}^{+}, \quad 1 \leq i \leq n .
$$

$$
r_{18} \equiv\left[e_{i}\right]_{1}^{0} \stackrel{0.5}{\longrightarrow}[\#]_{1}^{+}, \quad 1 \leq i \leq n .
$$

Configuration 2 is obtained by applying the rules belonging to the mortality module in configuration 1 (see Fig. 10).

- Feeding and density regulation module (1)

Checking whether maximum density has been reached.

$$
r_{19} \equiv\left(\left[D_{i}^{d_{k, i, 1}} a_{i}^{d_{k, i, 1}-d_{k, i, 2}} \rightarrow \#\right]_{1}^{+}\right)_{e_{k}}, \quad\left\{\begin{array}{l}
1 \leq k \leq 2, \\
1 \leq i \leq n .
\end{array}\right.
$$

Preparing animals for the feeding process.

$$
r_{20} \equiv\left[Y_{i, j, y} \rightarrow Z_{i, j, y}\right]_{1}^{+}, \quad\left\{\begin{array}{l}
1 \leq i \leq n, \\
0 \leq j<g_{i, 6} \\
1 \leq y \leq T
\end{array}\right.
$$

P system synchronizer.

$r_{21} \equiv\left[R_{0}\right]_{1}^{+} \rightarrow\left[R_{1}\right]_{0}^{0}$.

After applying the rules $r_{19}, r_{20}$ and $r_{21}$ we obtain configuration 3 (see Fig. 10).

The following rule is used in order to synchronize the model.

$r_{22} \equiv\left[R_{i} \rightarrow R_{i+1}\right]_{0}^{0}, 1 \leq i \leq 6$

Feeding process for animal with physical space in the ecosystem (object $a_{i}$ ).

$$
\begin{aligned}
& r_{23} \equiv\left[Z_{i, j, y} a_{i} B^{f_{i, 5}} \cdot g_{i, 2} G^{f_{i, 6}} \cdot g_{i, 2} M^{f_{i, 7} \cdot g_{i, 2} S^{f_{i, 7}} \cdot g_{i, 2} \cdot\left(1-g_{i, 7}\right)}\right]_{1}^{+} \rightarrow\left[W_{i, j, y}\right]_{1}^{0}, \\
& \left\{\begin{array}{l}
1 \leq i \leq n, \\
0 \leq j \leq g_{i, 6} \\
1 \leq y \leq T
\end{array},\right. \\
& r_{24} \equiv\left[Z_{i, j, y} a_{i} B^{f_{i, 5}} \cdot g_{i, 2} G^{f_{i, 6}} \cdot g_{i, 2} M^{f_{i, 7}} \cdot g_{i, 2} \cdot\left(1-g_{i, 7}\right) S^{f_{i, 7}} \cdot g_{i, 2} \cdot g_{i, 7}\right]_{1}^{+} \rightarrow\left[W_{i, j, y}\right]_{1}^{0}, \\
& \left\{\begin{array}{l}
1 \leq i \leq n, \\
0 \leq j \leq g_{i, 6}, \\
1 \leq y \leq T .
\end{array}\right.
\end{aligned}
$$

The configuration that is obtained after application of this rule is configuration 4 (see Fig. 10).

- Change in the environment module

Change in environment.

1 step: object coming out to the environment.

$r_{25} \equiv\left[Z_{i, j, y}\right]_{1}^{0} \rightarrow Z_{i, j, y}[]_{1}^{0}, \quad\left\{\begin{array}{l}1 \leq i \leq n, \\ 0 \leq j \leq g_{i, 6} \\ 1 \leq y \leq T\end{array}\right.$

See configuration 5 (Fig. 10).

2 step: objects are sending to the environment.

$r_{26} \equiv\left[Z_{i, j, y}\right]_{0}^{0} \rightarrow Z_{i, j, y}[]_{0}^{0},\left\{\begin{array}{l}1 \leq i \leq n, \\ 0 \leq j \leq g_{i, 6}, \\ 1 \leq y \leq T\end{array}\right.$

See configuration 6 (Fig. 10).

3 step: the object changes the environment

$r e_{1} \equiv\left(Z_{i, j, y}\right)_{e_{k}} \stackrel{p(i, k, v)}{\longrightarrow}\left(Z_{i, j, y}^{\prime}\right)_{e_{v}},\left\{\begin{array}{l}1 \leq i \leq n, \\ 0 \leq j \leq g_{i, 6}, \\ 1 \leq y \leq T, \\ 1 \leq k, v \leq 2 .\end{array}\right.$

See configuration 7 (Fig. 10).

4 step: the object enters the environment

$r_{27} \equiv Z_{i, j, y}^{\prime}[]_{0}^{0} \rightarrow\left[Z_{i, j, y}^{\prime}\right]_{0}^{0},\left\{\begin{array}{l}1 \leq i \leq n, \\ 0 \leq j \leq g_{i, 6} \\ 1 \leq y \leq T\end{array}\right.$

See configuration 8 (Fig. 10).

5 step: the object enters the membrane 1 .

$r_{28} \equiv Z_{i, j, y}^{\prime}[]_{1}^{0} \rightarrow\left[Z_{i, j, y}^{\prime}\right]_{1}^{0}, \quad\left\{\begin{array}{l}1 \leq i \leq n, \\ 0 \leq j \leq g_{i, 6} \\ 1 \leq y \leq T\end{array}\right.$

See configuration 9 (Fig. 10).

- Feeding and density regulation module (2) 

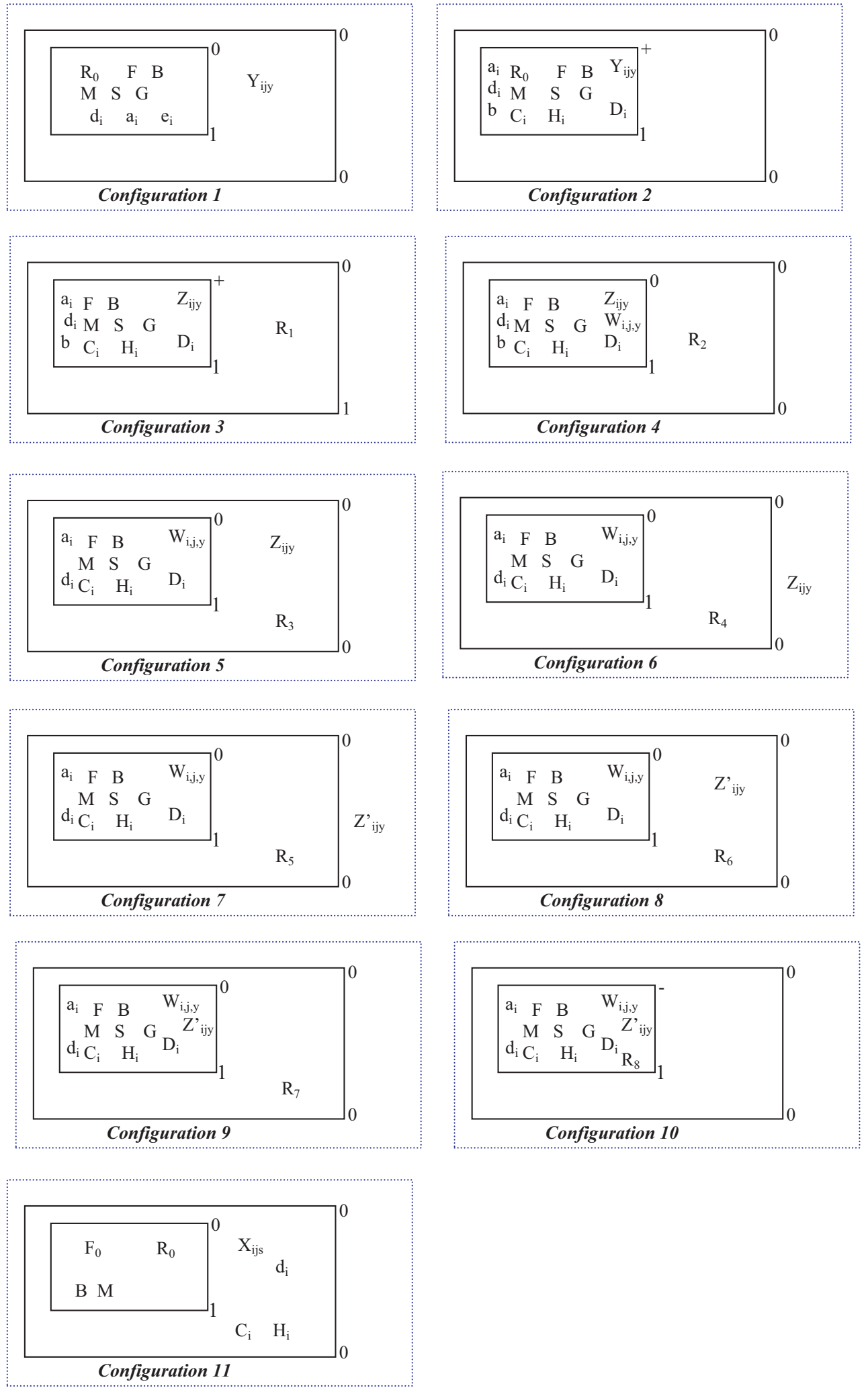

Fig. 10. Eleven configurations that form a loop (year, see also Fig. 6) of the model proposed for the study of an ecosystem occupied by avian scavengers. 
Feeding and determination of the physical space available for animals coming from another environment.

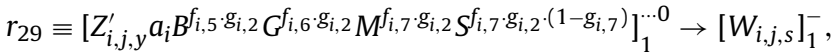

$$
\begin{aligned}
& \left\{\begin{array}{l}
1 \leq i \leq n, \\
0 \leq j \leq g_{i, 6}, \\
1 \leq y \leq T .
\end{array}\right. \\
& r_{30} \equiv\left[Z_{i, j, y}^{\prime} a_{i} B^{f_{i, 5}} \cdot g_{i, 2} G^{f_{i, 6}} \cdot g_{i, 2} M^{f_{i, 7}} \cdot g_{i, 2} \cdot\left(1-g_{i, 7}\right) S_{i, 7} \cdot g_{i, 2} \cdot g_{i, 7}\right]_{1}^{0} \rightarrow\left[W_{i, j, y}\right]_{1}^{-}, \\
& \left\{\begin{array}{l}
1 \leq i \leq n, \\
0 \leq j \leq g_{i, 6}, \\
1 \leq y \leq T .
\end{array}\right.
\end{aligned}
$$

$r_{31} \equiv R_{7}[]_{1}^{0} \rightarrow\left[R_{8}\right]_{1}^{-}$.

See configuration 10 (Fig. 10).

- Updating module

Preparations for the beginning of a new cycle, new simulation year.

The objects associated with the animals involved in objects $X$; they are prepared to begin the new cycle.

$r_{32} \equiv\left[W_{i, j, y}\right]_{1}^{-} \rightarrow X_{i, j+1, y+1}[]_{1}^{0}, \quad\left\{\begin{array}{l}1 \leq i \leq n, \\ 0 \leq j \leq g_{i, 6} \\ 1 \leq y \leq T\end{array}\right.$

The objects are initialized.

$r_{33} \equiv\left[R_{8}\right]_{1}^{-} \rightarrow\left[R_{0}\right]_{1}^{0}$.

$r_{34} \equiv[F]_{1}^{-} \rightarrow\left[F_{0}\right]_{1}^{0}$

Objects created during the process must be eliminated.

$$
\begin{aligned}
& r_{35} \equiv[G]_{1}^{-} \rightarrow[]_{1}^{0} . \\
& r_{36} \equiv[M]_{1}^{-} \rightarrow[]_{1}^{0} . \\
& r_{37} \equiv[S]_{1}^{-} \rightarrow[]_{1}^{0} . \\
& r_{38} \equiv[B]_{1}^{-} \rightarrow[]_{1}^{0} . \\
& r_{39} \equiv\left[a_{i}\right]_{1}^{-} \rightarrow[]_{1}^{0}, \quad 1 \leq i \leq n . \\
& r_{40} \equiv\left[d_{i}\right]_{1}^{-} \rightarrow d_{i}[]_{1}^{0}, \quad 1 \leq i \leq n . \\
& r_{41} \equiv\left[D_{i}\right]_{1}^{-} \rightarrow[]_{1}^{0}, \quad 1 \leq i \leq n .
\end{aligned}
$$

It is necessary to count the objects $H$ and $C$ so they are sending to skin membrane.

$$
\begin{aligned}
& r_{42} \equiv\left[H_{i}\right]_{1}^{-} \rightarrow H_{i}[]_{1}^{0}, \quad 1 \leq i \leq n . \\
& r_{43} \equiv\left[C_{i}\right]_{1}^{-} \rightarrow C_{i}[]_{1}^{0}, 1 \leq i \leq n .
\end{aligned}
$$

$r_{44} \equiv[b \rightarrow \#]_{1}^{0}$

The animal without enough food or space dies and leaves biomass.

$$
r_{45} \equiv\left[Z_{i, j, y}^{\prime}\right]_{1}^{-} \stackrel{g_{i, 1}}{\longrightarrow}\left[H_{i}^{f_{i, 1} \cdot f_{i, 8}} C_{i}^{f_{i, 2} \cdot f_{i, 9}} B_{i}^{f_{i, 1} \cdot f_{i, 8}} M_{i}^{f_{i, 2} \cdot f_{i, 9}}\right]_{1}^{0}, \quad\left\{\begin{array}{l}
1 \leq i \leq n, \\
0 \leq j<g_{i, 3}, \\
1 \leq y \leq T .
\end{array}\right.
$$

$r_{46} \equiv\left[Z_{i, j, y}^{\prime}\right]_{1}^{-} \stackrel{g_{i, 1}}{\longrightarrow}\left[H_{i}^{f_{i, 3} \cdot f_{i, 8}} C_{i}^{f_{i, 4} \cdot f_{i, 9}} B_{i}^{f_{i, 3} \cdot f_{i, 8}} M_{i}^{f_{i, 4} \cdot f_{i, 9}}\right]_{1}^{0}, \quad\left\{\begin{array}{l}1 \leq i \leq n, \\ g_{i, 3} \leq j \leq g_{i, 6}, \\ 1 \leq y \leq T .\end{array}\right.$

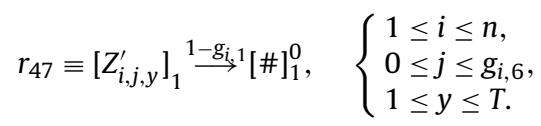

See configuration 11 (Fig. 10).

These rules overlap with the first rules, loop beginning.

$$
\begin{array}{ll}
r_{48} \equiv\left[H_{i} \rightarrow \#\right]_{0}^{0}, & 1 \leq i \leq n . \\
r_{49} \equiv\left[C_{i} \rightarrow \#\right]_{0}^{0}, & 1 \leq i \leq n .
\end{array}
$$

\section{References}

Bascompte, J., 2009. Disentangling the Web of Life. Science 325, 418-419. Bascompte, J., Melián, C.J., Sala, E., 2005. Interaction strength combinations and the overfishing of a marine food web. Proc. Natl. Acad. Sci. 102, 5443-5447.

Blasco, I., Olleta, J.L., San Juan, L., Revilla, R., 1992. The Pyrenean breed: some productive trials and the interest on its conservation in mountains areas. Arch. Zoot. 41 (extra), 549-553.

Brook, B.W., Cannon, J.R., Lacy, R.C., Mirande, C., Frankham, R., 1999. Comparison of the population viability analysis packages GAPPS, INMAT, RAMAS and VORTEX for the whooping crane (Grus americana). Anim. Conserv, 2, 23-31.

Cardona, M., Colomer, M.A., Pérez Hurtado, I., Pérez Jiménez, M.J., Sanuy, D., Margalida, A., 2009. Modelling ecosystems using P Systems: the Bearded Vulture, a case study. Lecture Notes Comp. Sci. 5391, 137-156.

Cardona, M., Colomer, M.A., Margalida, A., Pérez-Hurtado, I., Pérez-Jiménez, M.J. Sanuy, D., 2010a. A P-System based model of an ecosystem of some scavenger birds. Lecture Notes Comp. Sci. 5957, 182-195.

Cardona, M., Colomer, M.A., Margalida, A., Palau, A., Pérez-Hurtado, I., Pérez-Jiménez M.J., Sanuy, D., 2010b. A computational modeling for real ecosystems based on P Systems. Nat. Comput., 10.1007/s11047-010-9191-3.

Casasús, I., Villalba, D., Blanch, M., Sanz, A., Ferrer, R., Revilla, R., 1999. Cattle and sheep performance during summer grazing on high mountain ranges in extensive production systems. Options Méditerranéennes 27, 235-244.

Ciobanu, G., Păun, G., Perez-Jimenez, M.J. (Eds.), 2006. Applications of Membrane. Computing. Springer, Berlin.

Chapron, G., Wielgus, R., Quenette, P.Y., Camarra, J.J., 2009. Diagnosing mechanisms of decline and planning for recovery of an endangered brown bear (Ursus arctos) population. PLoS ONE 4, e7568.

Da Silva Peixoto, M., Carvalho de Barros, L., Bassanezi, R., 2008. Predator-prey fuzzy model. Ecol. Model. 214, 39-44.

Donázar, J.A., 1993. Los buitres ibéricos: biología y conservación. In: Reyero, J.M. (Ed.) Madrid.

Donázar, J.A., Margalida, A., Carrete, M., Sánchez-Zapata, J.A., 2009. Too sanitary for vultures. Science 326, 664.

Fulton, E.A., Parslow, J.S., Smith, A.D.M., Johnson, C.R., 2004. Biogeochemical marine ecosystem models. ii. The effect of physiological detail on model performance. Ecol. Model. 173, 371-406.

Fulton, E.A., Smith, A.D.M., Johnson, C.R., 2003. Mortality and predation in ecosystem models: is it important how these are expressed? Ecol. Model. 169, 157-178.

García, A., 2008. Dinámica reciente de los sistemas de vacuno en el Pirineo Central y evaluación de sus posibilidades de adaptación al entorno socio-económico. PhD University of Zaragoza, Zaragoza.

Gillespie, D.T., 1976. A general method for numerically simulating the stochastic time evolution of coupled chemical reactions. J. Comp. Phys. 22, 403-434.

Gillespie, D.T., 1977. Exact stochastic simulation of coupled chemical reactions. J. Phys. Chem. 81, 2340-2361. 
Grande, J.M. 2006. Factores limitantes, antrópicos y naturals de poblaciones de aves carroñeras: el caso del alimoche (Neoprhon percnopterus) en el Valle del Ebro. PhD thesis. University of Sevilla, Sevilla.

Grande, J.M., Serrano, D., Tavecchia, G., Carrete, M., Ceballos, O., Díaz-Delgado, R. Tella, J.L., Donázar, J.A., 2009. Survival in a long-lived territorial migrant: effects of life-history traits and ecological conditions in wintering and breeding areas. Oikos 118, 580-590.

Lawrie, J., Hearne, J., 2008. A method aggregating state variables in large ecosystem models. Math. Comput. Simul. 79, 368-378.

Litvaitis, J.A., Villafuerte, R., 1996. Intraguild predation, mesopredator release, and prey stability. Conserv. Biol. 10, 676-677.

Le Gouar, P., Robert, A., Choisy, J.P., Henriquet, S., Lecuyer, P., Tessier, Ch., Sarrazin, F. 2008. Roles of survival and dispersal in reintroduction success of griffon vulture (Gyps fulvus). Ecol. Appl. 18, 859-872.

Margalida, A., Donázar, J.A., Carrete, M., Sánchez-Zapata, J.A., 2010. Sanitary versus environmental policies: fitting together two pieces of the puzzle of European vulture conservation. J. Appl. Ecol. 47, 931-935.

Margalida, A., Garcia, D., Bertran, J., Heredia, R., 2003. Breeding biology and success of the bearded vulture Gypaetus barbatus in the eastern Pyrenees. Ibis 145 244-252.

Margalida, A., Bertran, J., Boudet, J., 2005. Assessing diet of nestling Bearded Vultures: a comparison between direct observation methods. J. Field Ornithol. 76, 4045.

Margalida, A., Bertran, J., Heredia, R., 2009. Diet and food preferences of the endangered Bearded vulture Gypaetus barbatus: a basis for their conservation. Ibis 151 235-243.

Meretsky, V.J., Snyder, N.F.R., Beissinger, S.R., Clendenen, D.A., Wiley, J.W., 2000 Demography of the California Condor: implications for reestablishment. Conserv. Biol. 14, 957-967.

Miehls, A.L.J., Mason, D.M., Frank, K.A., Krause, A.E., Peacor, S.D., William, W., Taylor W.W., 2009. Invasive species impacts on ecosystem structure and function: comparison of the Bay of Quinte, Canada, and Oneida Lake, USA, before and alter zebra mussel invasión. Ecol. Model. 220, 3182-3193.
Montserrat, P., Villar, L., 2007. Ecología y gestión pastoral en el Pirineo. Una perspectiva de medio siglo. Pirineos 162, 89-107.

Morales, Y., Weber, L.J., Mynett, A.E., Newton, T.J., 2006. Mussel Dynamics Model a hydroinformatics tool for analyzing the effects of different stressors on the dynamics of freshwater mussel communities. Ecol. Model. 197, 448-460.

Mullon, G., Cury, P., Shannon, L., 2004. Viability model of trophic interactions in marine ecosystems. Nat. Res. Model. 17, 71-102.

Murray, J.D., 2002. Mathematical Biology: An Introduction. Springer-Verlag, New York.

Olea, P., Mateo-Tomás, P., 2009. The role of traditional farming practices in ecosystem conservation: the case of transhumance and vultures. Biol. Conserv. 142 1844-1853.

Oro, D., Margalida, A., Carrete, M., Heredia, R., Donázar, J.A., 2008. Testing the goodness of supplementary feeding to enhance population viability in an endangered vulture. PLoS One 3, e4084.

Ortega, E., Mañosa, S., Margalida, A., Sánchez, R., Oria, J., González, L.M., 2009. A demographic description of the recovery of the Vulnerable Spanish imperial eagle Aquila adalberti. Oryx 43, 113-121.

Păun, G., 1998. Computing with membranes. J. Comput. Syst. Sci. 61, 108-143.

Păun, G., Rozenberg, G., Salomaa, A. (Eds.), 2010. The Oxford Handbook of Membrane Computing. Oxford Univ. Press, Oxford

Polis, G.A., Holt, R.D., 1992. Intraguild predation: the dynamics of complex trophic interactions. Trends Ecol. Evol. 7, 151-154.

Roigé, X., 1995. Pirineo Catalán. Cuadernos de la trashumancia № 13. ICONA, Madrid.

Russell, J.C., Lecomte, V., Dumont, Y., Le Corre, M., 2009. Intraguild predation and mesopredator release effect on long-lived prey. Ecol. Model. 220, 10981104.

Sakanoue, S., 2007. Extended logistic model for growth of single-species populations Ecol. Model. 205, 159-168.

Sakanoue, S., 2009. A resource-based approach to modelling the dynamics of interacting populations. Ecol. Model. 220, 1383-1394.

Ulanowicz, R.E., 2004. Quantitative methods for ecological network análisis. Comput. Biol. Chem. 28, 321-339. 